\title{
Figure $\mathrm{S}_{3}$
}

\section{Synthesis of Analog 3}

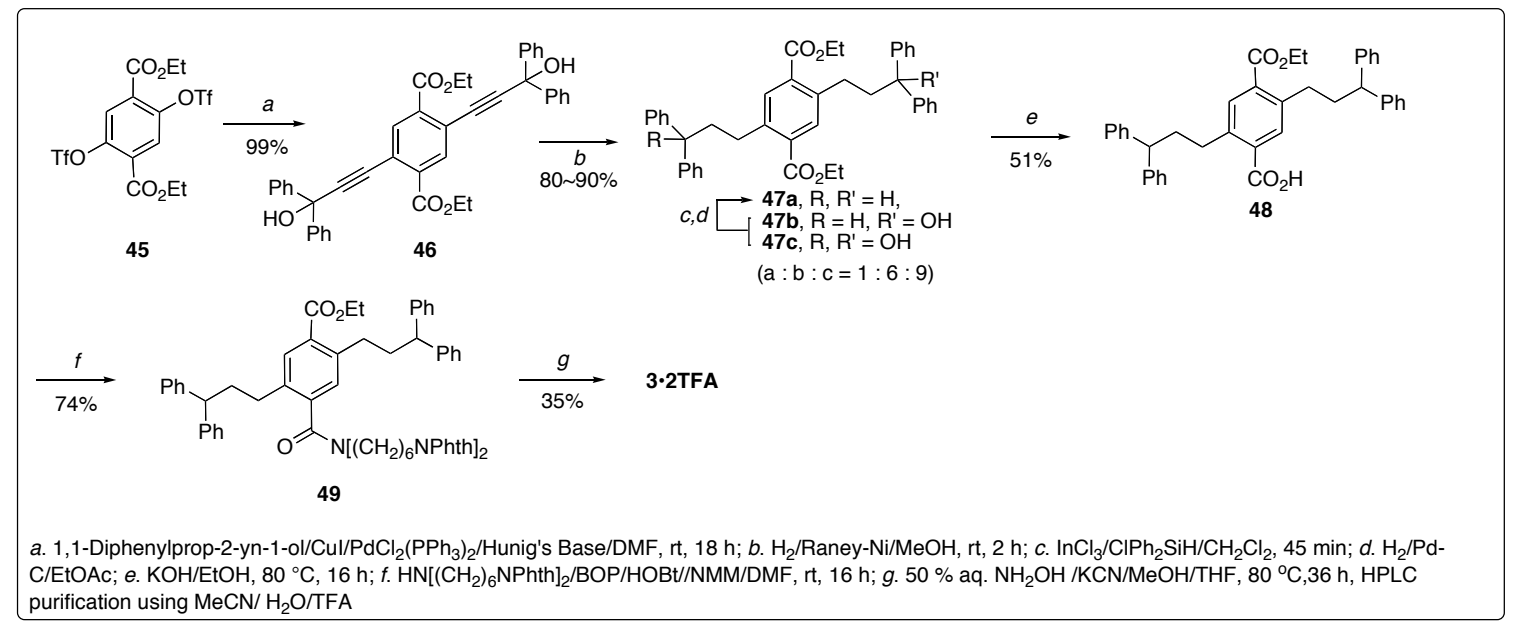

Diethyl 2,5-bis(3-hydroxy-3,3-diphenylprop-1-ynyl)terephthalate (46). The titled intermediate was synthesized following the known method[1], briefly, a solution of $\mathrm{CuI}(80 \mathrm{mg}, 0.42 \mathrm{mmol})$ and $\mathrm{PdCl}_{2}\left(\mathrm{PPh}_{3}\right)_{2}(0.15 \mathrm{~g}, 0.21 \mathrm{mmol})$ in anhydrous DMF $(6 \mathrm{~mL})$ and diisopropylethylamine (DIEA, $2.4 \mathrm{~mL}$ ) was degassed by purging with nitrogen for 15 minutes. To this was added

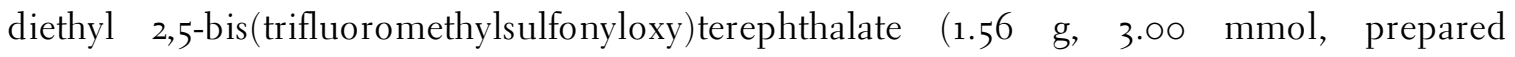
according to the literatre[2] and 1,1-diphenylprop-2-yn-1-ol $(1.37 \mathrm{~g}, 6.60 \mathrm{mmol})$ at room temperature under nitrogen. After 17 hours stirring at room temperature, the reaction mixture was diluted with $\mathrm{H}_{2} \mathrm{O}(10 \mathrm{~mL})$, extracted with EtOAc $(3 \times 20 \mathrm{~mL})$. The organic layer was stirred with decolorizing carbon ( $2 \mathrm{~g}$ ) for 10 minutes, filtered through a Celite pad to give a red filtrate. The filtrated was concentrated and purified by recrystallization from (EtOAc-Hex) to afford $0.70 \mathrm{~g}$ of white needles. The mother liquor was concentrated and purified by MPLC (gradient Hex to EtOAc) to give additional $1.18 \mathrm{~g}$ of the product (total $1.88 \mathrm{~g}, 99 \%$ ). ${ }^{1} \mathrm{H}$ NMR $\left(\mathrm{CDCl}_{3}\right) \delta 8.11(\mathrm{~s}, 2 \mathrm{H}), 7.71-7.68(\mathrm{~m}, 8 \mathrm{H}), 7.37-7.34(\mathrm{~m}, 8 \mathrm{H}), 7.30-7.27\left(\mathrm{~m},{ }_{4} \mathrm{H}\right), 4.31(\mathrm{q}, J=$ $7.2 \mathrm{~Hz}, 4 \mathrm{H}), 3.38(\mathrm{br} \mathrm{s}, 2 \mathrm{H})$, and $1.29(\mathrm{t}, J=7.2 \mathrm{~Hz}, 6 \mathrm{H}) ;{ }^{13} \mathrm{C} \mathrm{NMR}\left(\mathrm{CDCl}_{3}\right) \delta 164.99,144.86$, $136.04,134.94,128.57,128.04,126.38,122.74,99.41,84.86,75.27,62.22$, and 14.42 . 
Diethyl 2,5-bis(3,3-diphenylpropyl)terephthalate (47a). Raney-Ni (0.5 mL, slurry in water) was washed with $\mathrm{MeOH}(3 \times 2 \mathrm{~mL})$, covered with $5 \mathrm{~mL}$ of $\mathrm{MeOH}$. To this was added compound 2 (140 $\mathrm{mg}, 0.22 \mathrm{mmol}$ ). The resulting mixture was stirred at room temperature for 2 hours under a balloon of hydrogen. The aliquot was taken using a pipette, and the catalyst was washed several times with EtOAc. The washings and the aliquot were combined, concentrated in vacuo, and then purified by MPLC (gradient Hex to EtOAc) to give a mixture of $47 \mathbf{a}, 47 \mathbf{b}$ and $47 \mathrm{c}$ at the ratio of $1(6 \mathrm{mg}): 6(39 \mathrm{mg}): 9(63 \mathrm{mg})$ (in 80-90\% yield). The ratio varies batch by batch, but za was always the least. $47 \mathrm{a}:{ }^{1} \mathrm{H} \mathrm{NMR}\left(400 \mathrm{MHz} \mathrm{CDCl}_{3}\right) \delta 7.59(\mathrm{~s}, 2 \mathrm{H}), 7 \cdot 30-7.16$ $(\mathrm{m}, 2 \mathrm{OH}), 4.29\left(\mathrm{q}, J=7.0 \mathrm{~Hz},{ }_{4} \mathrm{H}\right), 3.96(\mathrm{t}, J=7.6 \mathrm{~Hz}, 2 \mathrm{H}), 2.89-2.85\left(\mathrm{~m},{ }_{4} \mathrm{H}\right), 2.38-2.32(\mathrm{~m}$, $\left.{ }_{4} \mathrm{H}\right)$, and $1.33(\mathrm{t}, J=7.0 \mathrm{~Hz}, 6 \mathrm{H}) ;{ }^{13} \mathrm{C} \mathrm{NMR}\left(100 \mathrm{MHz}, \mathrm{CDCl}_{3}\right) \delta 167.48,144.96,141.08,133.11$, 133.01, 128.69, 128.14, 126.41, 61.40, 51.55, 37.62, 32.67, and 14.51. Diethyl 2-(3.3diphenylpropyl)-5-(3-hydroxy-3,3-diphenylpropyl)terephthalate $(47 \mathrm{~b}):{ }^{1} \mathrm{H}$ NMR $\left(\mathrm{CDCl}_{3}\right) \delta$ $7.65\left(\mathrm{~s},{ }_{1} \mathrm{H}\right), 7.62\left(\mathrm{~s},{ }_{1} \mathrm{H}\right), 7.52-7.50\left(\mathrm{~m},{ }_{4} \mathrm{H}\right), 7.34-7.19(\mathrm{~m}, 16 \mathrm{H}), 4.37-4.27(\mathrm{~m} 4 \mathrm{H}), 3.97(\mathrm{t}, J=$ $\left.7.8 \mathrm{~Hz},{ }_{1} \mathrm{H}\right), 3.39\left(\mathrm{br} \mathrm{s},{ }_{1} \mathrm{H}\right), 2.92-2.87\left(\mathrm{~m},{ }_{4} \mathrm{H}\right), 2.64-2.58(\mathrm{~m} 2 \mathrm{H}), 2.4 \mathrm{O}-2.34(\mathrm{~m}, 2 \mathrm{H}), 1.43^{-1.32}$ (m, 6H). Diethyl 2,5-bis(3-hydroxy-3,3-diphenylpropyl)terephthalate (47c): ${ }^{1} \mathrm{H} \mathrm{NMR}\left(\mathrm{CDCl}_{3}\right)$ $\delta 7.65(\mathrm{~s}, 2 \mathrm{H}), 7.51-7.49(\mathrm{~m}, 8 \mathrm{H}), 7.34-7.30(\mathrm{~m}, 8 \mathrm{H}), 7.24-7.20\left(\mathrm{~m},{ }_{4} \mathrm{H}\right), 4.32(\mathrm{q}, J=7.0 \mathrm{~Hz}$ $\left.{ }_{4} \mathrm{H}\right), 3.36(\mathrm{br} \mathrm{s}, 2 \mathrm{H}), 2.90-2.86\left(\mathrm{~m},{ }_{4} \mathrm{H}\right), 2.63-2.58\left(\mathrm{~m},{ }_{4} \mathrm{H}\right)$, and $1.36(\mathrm{t}, J=7.0 \mathrm{~Hz}, 6 \mathrm{H}) ;{ }^{13} \mathrm{C}$ $\mathrm{NMR}\left(\mathrm{CDCl}_{3}\right) \delta 167.28,147.34,142.04,133.53,132.66,128.34,126.94,126.27,61.72,44.13,28.91$, and 14.53 .

Both $47 \mathrm{~b}$ and $47 \mathrm{c}$ were (individually or as a mixture of the two) treated with $\mathrm{InCl}_{3}(5 \mathrm{~mol} \%)$ in the presence of Chlorodiphenylsilane $\left(\mathrm{ClPh}_{2} \mathrm{SiH}, 2\right.$ eq) in $\mathrm{CH}_{2} \mathrm{Cl}_{2}$ at room temperature for $30-$ 45 minutes to dehydrate and give mono- and di-olefinic intermediate, respectively. The conditions were applied following the literature procedure.[3] The mono-olefinic intermediate (diethyl 2-(3,3-diphenylallyl)-5-(3,3-diphenylpropyl)terephthalate): ${ }^{1} \mathrm{H}$ NMR $\left(\mathrm{CDCl}_{3}\right) \delta_{7} .67$ (d, $\left.J=1.4 \mathrm{~Hz},{ }_{1} \mathrm{H}\right), 7.63\left(\mathrm{~d}, J=1.6 \mathrm{~Hz},{ }_{1} \mathrm{H}\right), 7.43-7.17(\mathrm{~m}, 2 \mathrm{OH}), 6.26\left(\mathrm{dt}, J=1.6,7.2 \mathrm{~Hz},{ }_{1} \mathrm{H}\right)$, 
$4.37-4.27\left(\mathrm{~m},{ }_{4} \mathrm{H}\right), 3.98\left(\mathrm{t}, J=7.8 \mathrm{~Hz},{ }_{1} \mathrm{H}\right), 3.79(\mathrm{~d}, J=7.2 \mathrm{~Hz}, 2 \mathrm{H}), 2.92-2.88(\mathrm{~m}, 2 \mathrm{H}), 2.4 \mathrm{O}-$ $2.35(\mathrm{~m}, 2 \mathrm{H})$, and $1.39-1.32(\mathrm{~m}, 6 \mathrm{H}) ;{ }^{13} \mathrm{C} \mathrm{NMR}\left(\mathrm{CDCl}_{3}\right) \delta_{167.48}, 167.34,144.97,142.91,142.65$, $141.38,140.04,139.71,133.15,133.08,132.95,132.89,130.19,128.71,128.55,128.33,128.16,127.68$, $127.59,127.42,127.30,126.43,61.52,61.38,51.55,37.62,34.02,32.68,14.53$, and 14.49. The diolefinic intermediate (diethyl 2,5-bis(3,3-diphenylallyl)terephthalate): ${ }^{1} \mathrm{H} \mathrm{NMR}\left(\mathrm{CDCl}_{3}\right) \delta 7.69$ $(\mathrm{s}, 2 \mathrm{H}), 7.43-7.22(\mathrm{~m}, 2 \mathrm{OH}), 6.27(\mathrm{t}, J=7.2 \mathrm{~Hz}, 2 \mathrm{H}), 4.33(\mathrm{q}, J=7.0 \mathrm{~Hz}, 4 \mathrm{H}), 3.81(\mathrm{~d}, J=7.2$ $\left.\mathrm{Hz},{ }_{4} \mathrm{H}\right), 1.36(\mathrm{t}, J=7.0 \mathrm{~Hz}, 6 \mathrm{H}) ;{ }^{13} \mathrm{C} \mathrm{NMR}\left(\mathrm{CDCl}_{3}\right) \delta 167.36,142.94,142.63,140.04,139.98$ $134.57,133.06,132.84,130.20,128.56,128.33,127.63,127.59,127.43,127.31,61.49,33.99$, and 14.49. These intermediates were stirred under hydrogen (balloon) in EtOAc to give za in 89\% yield over two steps.

2,5-Bis(3,3-diphenylpropyl)-4-(ethoxycarbonyl)benzoic acid (48). The compounds 47 a (173 $\mathrm{mg}, 0.28 \mathrm{mmol}$ ) was dissolved in $\mathrm{EtOH}(5 \mathrm{~mL})$, to this was added potassium hydroxide $(32 \mathrm{mg}$, 2.0 eq), and then heated at $80{ }^{\circ} \mathrm{C}$ for 14 hours. After cooling to room temperature, the solvent was removed in vacuo, the residue diluted with $\mathrm{H}_{2} \mathrm{O}(5 \mathrm{~mL})$, acidified with $1 \mathrm{~N} \mathrm{HCl}$ to $\mathrm{pH} 3$, and then extracted with EtOAc. The organic layer was washed with brine, dried over $\mathrm{MgSO}_{4}$, concentrated in vacuo, and then purified by MPLC (gradient Hex to EtOAc) to afford the title compound (84 mg, 51\%). ${ }^{1} \mathrm{H}$ NMR $\left(\mathrm{CDCl}_{3}\right) \delta 7.72\left(\mathrm{~s},{ }_{1} \mathrm{H}\right), 7.53(\mathrm{~s}, 1 \mathrm{H}), 7 \cdot 72-7.03(\mathrm{~m}, 2 \mathrm{OH})$, $4.33-4.30(\mathrm{~m}, 2 \mathrm{H}), 3.84-3.76(\mathrm{~m}, 2 \mathrm{H}), 2.82-2.74\left(\mathrm{~m},{ }_{4} \mathrm{H}\right), 2.21-2.06\left(\mathrm{~m},{ }_{4} \mathrm{H}\right)$, and $1.35\left(\mathrm{~m},{ }_{3} \mathrm{H}\right)$.

\section{Ethyl 4-(bis(6-(1,3-dioxoisoindolin-2-yl)hexyl)carbamoyl)-2,5-bis(3,3-diphenylpropyl)benzoate}

(49). To a stirred solution of compound $48\left(18.9 \mathrm{mg}, 3.24 \times 10^{-5} \mathrm{~mol}\right), \mathbf{1 4} \times\left(15.4 \mathrm{mg}, 3.24 \times 10^{-5}\right.$ $\mathrm{mol}), \operatorname{BOP}\left(21.5 \mathrm{mg}, 4.87 \times 10^{-5} \mathrm{~mol}\right)$, and $\operatorname{HOBt}\left(6.6 \mathrm{mg}, 4.87 \times 10^{-5} \mathrm{~mol}\right)$ in $1 \mathrm{~mL} \mathrm{DMF}$ was added NMM $\left(5.3 \mu \mathrm{L}, 4.87 \times 10^{-5} \mathrm{~mol}\right)$ at room temperature. After stirring overnight at the same temperature, diluted with $\mathrm{H}_{2} \mathrm{O}(2 \mathrm{~mL})$, extracted with EtOAc $(3 \times 3 \mathrm{~mL})$. The extracts were combined, washed with brine, dried over $\mathrm{MgSO}_{4}$, concentrated, and then purified by 
MPLC (gradient Hex to EtOAc) to afford $49(0.025 \mathrm{~g}, 74 \%) .{ }^{1} \mathrm{H} \mathrm{NMR}\left(\mathrm{CDCl}_{3}\right) \delta 7.85-7.81$ (m, $\left.{ }_{4} \mathrm{H}\right), 7.71-7.69\left(\mathrm{~m},{ }_{4} \mathrm{H}\right), 7.66\left(\mathrm{~s},{ }_{1} \mathrm{H}\right), 7.26-7.22(\mathrm{~m}, 16 \mathrm{H}), 7.15-7.12\left(\mathrm{~m},{ }_{4} \mathrm{H}\right), 6.92\left(\mathrm{~s},{ }_{1} \mathrm{H}\right), 4.27$ $(\mathrm{q}, J=7.0 \mathrm{~Hz}, 2 \mathrm{H}), 3.96\left(\mathrm{t}, J=7.7 \mathrm{~Hz},{ }_{1} \mathrm{H}\right), 3.91\left(\mathrm{t}, J=7.7 \mathrm{~Hz},{ }_{1} \mathrm{H}\right), 3.71(\mathrm{t}, J=7.2 \mathrm{~Hz}, 2 \mathrm{H})$, $3.52(\mathrm{t}, J=7.2 \mathrm{~Hz}, 2 \mathrm{H}), 3.48(\mathrm{~m}, 1 \mathrm{H}), 3.22-3.17(\mathrm{~m}, 1 \mathrm{H}), 2.96-2.72(\mathrm{~m}, 4 \mathrm{H}), 2.47(\mathrm{~m}, 1 \mathrm{H}), 2.35-$ $2.25(\mathrm{~m}, 3 \mathrm{H}), 1.73-1.69(\mathrm{~m}, 2 \mathrm{H}), 1.52-1.30\left(\mathrm{~m},{ }_{15} \mathrm{H}\right)$, and $1.12-0.97\left(\mathrm{~m},{ }_{4} \mathrm{H}\right) ;{ }^{13} \mathrm{C} \mathrm{NMR}\left(\mathrm{CDCl}_{3}\right)$ $\delta 170.17,168.69,168.57,167.61,145.05,144.98,144.48,141.83,140.29,136.06,134.13,132.38$, $132.32,131.76,130.31,128.69,128.67,128.46,128.17,128.07,128.02,126.41,126.37,123.42,123.40$, $61.22,51.78,51.55,48.51,44.40,38.13,37.85,36.63,32.96,31.43,28.82,28.55,27.32,26.96,26.84$, $26.57,26.43$, and 14.55 .

$N^{1}, N^{1}$-Bis(6-aminohexyl)-2,5-bis(3,3-diphenylpropyl)-N4-hydroxyterephthalamide (3). To a stirred solution of the compound $5\left(25 \mathrm{mg}, 2.40 \times 10^{-5} \mathrm{~mol}\right)$ in $0.5 \mathrm{~mL}$ each of THF and $\mathrm{MeOH}$ was added 2 crystals of $\mathrm{KCN}$ at room temperature. The mixture was heated at $80{ }^{\circ} \mathrm{C}$ for 36 hours. After cooling to room temperature, the mixture was concentrated, purified by HPLC to give 3. 2TFA (8.4 mg, 35\%). Analytical retention time was 14.18 minutes for the product. ${ }^{1} \mathrm{H}$ NMR $\left(\mathrm{DMSO}_{6}\right) \delta 13.00($ brs, $2 \mathrm{H}), 7.70-7.62(\mathrm{~m}, 2 \mathrm{H}), 7.62(\mathrm{~s}, 1 \mathrm{H}), 7.27-7.15$ (m, $20 \mathrm{H}), 6.92(\mathrm{~s}, 1 \mathrm{H}), 3.96-3.90(\mathrm{~m} 2 \mathrm{H}), 3.54(\mathrm{brs}, 6 \mathrm{H}), 3.42-3.40(\mathrm{~m}, 2 \mathrm{H}), 3.15(\mathrm{~m}, 2 \mathrm{H}), 2.80-$ $2.74\left(\mathrm{~m},{ }_{4} \mathrm{H}\right), 2.57-2.54(\mathrm{~m}, 2 \mathrm{H}), 2.36-2.21(\mathrm{~m}, 6 \mathrm{H}), 1.56-1.52(\mathrm{~m}, 2 \mathrm{H}), 1.4 \mathrm{O}-1.22(\mathrm{~m}, 10 \mathrm{H})$, and $0.97-0.92\left(\mathrm{~m},{ }_{4} \mathrm{H}\right) ;{ }^{13} \mathrm{C}$ NMR (DMSO-d 6 ) $\delta 169.53,169.13,145.55,141.29,136.06,131.19$, $129.13,128.23,126.80,51.49,48.44,44.29,43.64,39.25,37.61,36.47,32.61,31.38,28.61,27.78$, $27.48,27 \cdot 32,26.77,26.30,26.27,26.06$, and 23.33 .

\section{References}

1. Sorensen JK, Vestergaard M, Kadziola A, Kilsa K, Nielsen MB (2006) Synthesis of Oligo(phenyleneethylene)-Tetrathiafulvalene Cruciforms for Molecular Electronics. Organic Letters 8: 1173-1176. 
2. Zhang Q, Shi C, Zhang H-R, Wang KK (2000) Synthesis of 6H-Indolo[2,3b] $[1,6]$ naphthyridines and Related Compounds as the 5-Aza Analogues of Ellipticine Alkaloids. The Journal of Organic Chemistry 65: 7977-7983.

3. Yasuda M, Onishi Y, Ueba M, Miyai T, Baba A (2001) Direct Reduction of Alcohols: Highly Chemoselective Reducing System for Secondary or Tertiary Alcohols Using Chlorodiphenylsilane with a Catalytic Amount of Indium Trichloride. The Journal of Organic Chemistry 66: 7741-7744. 